\title{
Clinical Application of Central Venous Catheter in Closed Thoracic Drainage After Minimally Invasive Surgery for Esophageal Cancer
}

\section{Yang Zhao}

General Hospital of Ningxia Medical University

\section{Zhixia Bai}

General Hospital of Ningxia Medical University

\section{Tao Wang}

General Hospital of Ningxia Medical University

Dong Song

General Hospital of Ningxia Medical University

\section{Tao Li ( $\sim$ litao2151@yeah.net )}

General Hospital of Ningxia Medical University https://orcid.org/0000-0003-4854-4579

Research article

Keywords: central venous catheter, esophageal cancer, minimally invasive surgery, thoracic drainage

Posted Date: November 2nd, 2020

DOI: https://doi.org/10.21203/rs.3.rs-99490/v1

License: (9) This work is licensed under a Creative Commons Attribution 4.0 International License. Read Full License 


\section{Clinical application of central venous catheter in closed thoracic drainage after minimally invasive surgery for esophageal cancer}

Yang Zhao ${ }^{1}$, Zhi-xia Bai ${ }^{2}$, Tao Wang ${ }^{1}$, Dong Song ${ }^{1}$, Tao Li $^{*}$

1 Department of Surgical Oncology II, General Hospital of Ningxia Medical University, Yinchuan 750004, Ningxia, China

2 Department of Anesthesiology, General Hospital of Ningxia Medical University, Yinchuan 750004, Ningxia, China

*Corresponding author: Tao $\mathrm{Li}$,

Department of Surgical Oncology II, General Hospital of Ningxia Medical University, Yinchuan 750004, Ningxia, China

Tel: $+86-951-6743770$

E-mail:litao2151@yeah.net.

Email addresses of all co-authors: zhaoyang2775@163.com, zx4073354@163.com, wangtao25522552@163.com, songdong2475@163.com

\section{[Abstract]}

Objective: To evaluate the clinical safety and practicability of the closed thoracic drainage device after esophageal cancer surgery, so as to reduce the postoperative pain and shorten the length of the hospital stay, speed up the postoperative recovery of the patients.

Methods: A retrospective analysis of patients who underwent minimally invasive surgery for esophageal cancer in the Second Department of Surgery, General Hospital of Ningxia Medical University from January 2017 to December 2019. Among them, 44 and 59 patients underwent central venous catheter thoracic drainage and traditional closed thoracic drainage, respectively. Recorded data included postoperative pleural drainage, postoperative extubation time, postoperative complications (postoperative encapsulated pleural effusion, postoperative subcutaneous emphysema, postoperative atelectasis, postoperative pneumothorax, postoperative pulmonary infection, postoperative anastomotic leakage), catheter re-insertion after extubation, postoperative hospital stay, postoperative drainage tube healing time, and postoperative pain score, etc.

Results: There was no statistical difference in the postoperative chest drainage (d1) $(547.72 \pm$ $64.66 \& 536.27 \pm 84.67 \mathrm{ml})$, postoperative extubation time $(7.61 \pm 0.92 \& 7.53 \pm 1.02 \mathrm{~min})$, or postoperative complications between the experimental group and the control group $(p>0.05)$. Postoperative thoracic drainage (d2-d5), postoperative hospital stay $(11.75 \pm 2.02 \& 13.03 \pm$ 1.29days), drainage tube healing time (5.64 $\pm 1.12 \& 14.02 \pm 1.32$ days), and postoperative pain scores were significantly different between the two groups of esophageal cancer patients $(p<$ $0.05)$.

Conclusion: The central venous catheter used for thoracic drainage is not only safe and reliable, 
but also greatly reduces postoperative pain and discomfort of patients, and accelerates postoperative recovery of patients. Therefore, it conforms to the concept of enhanced recovery after surgery, and is worthy of clinical application.

Keywords: central venous catheter, esophageal cancer, minimally invasive surgery, thoracic drainage

\section{Introduction}

Esophageal cancer is a common malignant tumor of the upper gastrointestinal $\operatorname{tract}^{[1]}$. Approximately 300,000 people die of esophageal cancer each year worldwide, and China is one of the areas with high incidence of esophageal cancer. Surgery is considered the first option for the treatment of esophageal cancer ${ }^{[2]}$. At present, minimally invasive techniques represented by thoracoscopy are widely used in the surgical treatment of esophageal cancer ${ }^{[3,}{ }^{4]}$. Although we are constantly pursuing the innovation of minimally invasive technology during surgery, the overall experience of patients after surgery are often overlooked. So far, thick closed thoracic drainage tubes are still being used after esophageal cancer surgery. Even though the material of the drainage tube has been updated from the previous harder plastic tube to a softer silicone tube, there is no significant improvement in pain and discomfort of the patients due to the thick postoperative drainage tube, which greatly reduces advantages of the minimally invasive surgery for esophageal cancer. This study aims to evaluate the clinical safety and practicability of the closed thoracic drainage device after esophageal cancer surgery, so as to reduce the postoperative pain and speed up the postoperative recovery of patients $\left.{ }^{[5,}{ }^{6}\right]$.

\section{Materials and methods}

\subsection{Patients and data collection}

Clinical data Retrospective analysis of Patients who underwent minimally invasive surgery for esophageal cancer in the Second Department of Oncology and Surgery, General Hospital of Ningxia Medical University from January 2017 to December 2019. Among them, 59 cases underwent traditional closed thoracic drainage and 44 cases underwent central venous catheter thoracic drainage in a total of 103 patients. This study was approved by the Regional Ethics Committee of our hospital and all patients signed informed consents.

\subsection{Inclusion and exclusion criteria}

Inclusion criteria: All patients underwent electronic gastroscopy and have clear results for pathological examination before surgery. Before the operation, chest computed tomography, bone scan and other related examinations were performed to confirm that the tumor had no obvious invasion and to exclude distant metastasis. Preoperative echocardiography, electrocardiogram, pulmonary function test were checked for the tolerance for the operation, and to make sure there is no absolute surgical contraindication. All operation methods were thoracolaparoscopic combined radical esophageal cancer surgery with additional tubular gastric left neck anastomosis.

Exclusion criteria: Patients with serious underlying diseases cannot tolerate surgery. Patients with signs of distant metastasis. Patients with tumor that has obviously invaded and involved the surrounding organs, with frequently enlarged mediastinal lymph nodes. Patients who turned to thoracotomy during surgery. Patients with past history of tuberculosis or other chest surgery. Those who received neoadjuvant radiotherapy and chemotherapy before surgery. 


\subsection{Drainage procedure}

All operation methods were thoracolaparoscopic combined radical esophageal cancer surgery with additional tubular gastric left neck anastomosis. Experimental group: After entering the thoracic cavity with a thick needle puncture needle in the ninth intercostal space of the right thorax, the single lumen central venous catheter was inserted, assisted with a guide wire(Fig 1A,C). The length of the catheter is about $7-10 \mathrm{~cm}$ in the thoracic cavity. The tip of the catheter is fixed at the posterior costal diaphragmatic angle. Be aware of the fix technique and ensure complete extraction. Control group: A 16F silicone tube was placed in the 8th intercostal operation hole in the midaxillary line(Fig 1B,D).

In the experimental group, the central venous catheter was connected with an anti-reflux drainage bag. The 24-hour thoracic drainage volume was less than $150 \mathrm{ml}$, and it was light yellow drainage. There was no obvious gas discharge from the thoracic cavity. The drainage bag could be removed if the upper gastrointestinal angiography showed no thoracic fistula. The control group was routinely connected to a water-sealed bottle, and the thoracic drainage was recorded daily. The data collection was consistent with the experimental group.

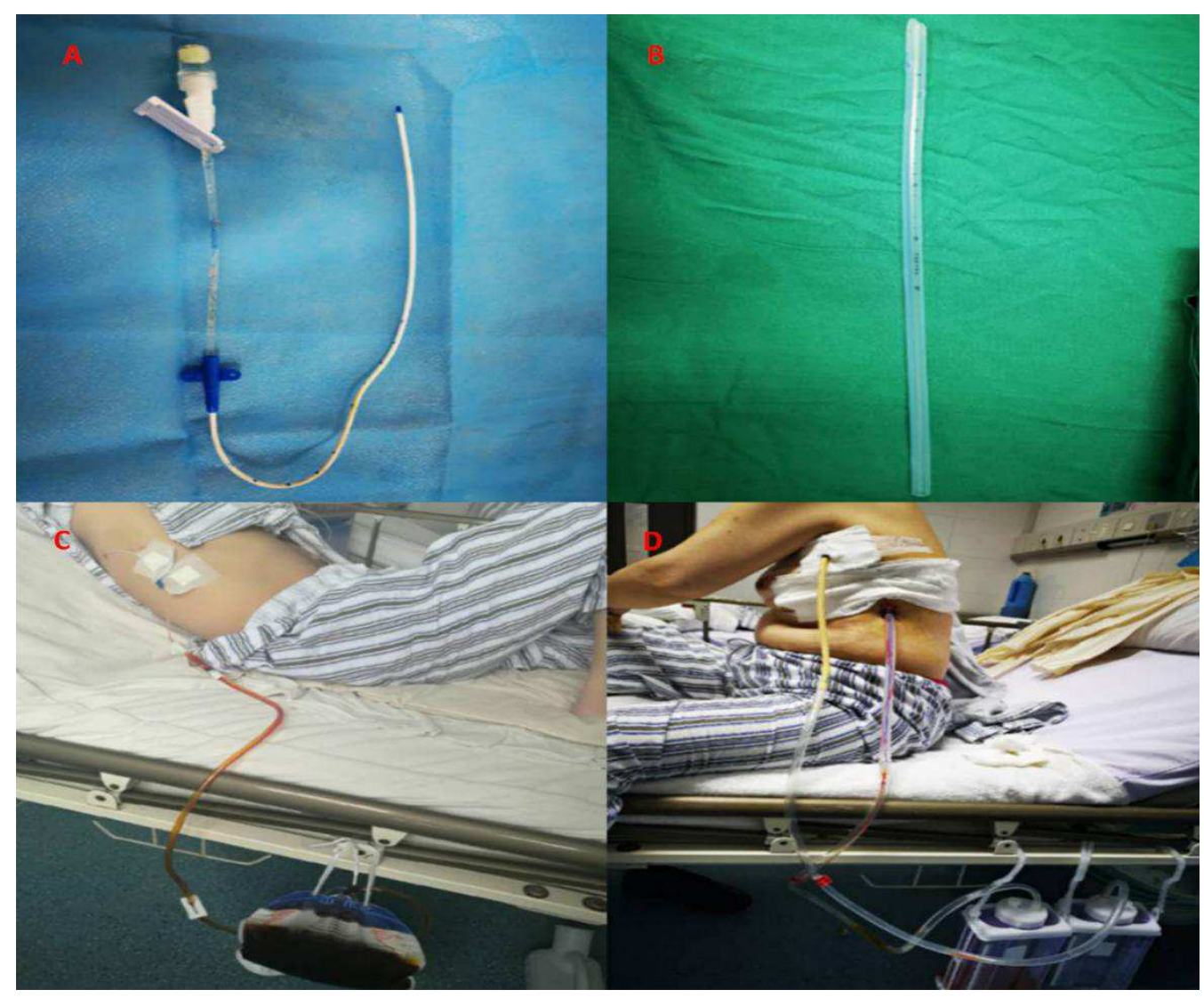

Fig1:A, The single lumen central venous catheter $(5 \mathrm{~F})$ with the diameter of $1.6 \mathrm{~mm} ; \mathrm{B}$, The silicone tube $(16 \mathrm{~F})$ with the diameter of $5.2 \mathrm{~mm}$; $\mathrm{C}, \mathrm{D}$, The single central venous catheter and the silicone tube drainage were used respectively after esophageal cancer surgery.

\subsection{Observation indicators}

The postoperative pleural drainage, postoperative extubation time, postoperative complications (postoperative encapsulated pleural effusion, postoperative subcutaneous emphysema, postoperative atelectasis, postoperative pleural effusion, postoperative pulmonary infection, 
postoperative anastomotic leakage), whether the catheter was re-inserted after extubation, postoperative hospital stay, postoperative drainage tube healing time, postoperative pain score, etc. were observed and recorded.

The most widely used evaluation form in the world, the Numerical Rating Scale, were employed to assess the postoperative pain: the numeric grading method uses $0-10$ to represent different degrees of pain. The grading standard of pain degree is: 0: no pain; 1-3: mild pain; 4-6: moderate pain; 7-10: severe pain. For moderate pain, oral clofenaceine tablets or paracetamol and oxycodone tablets can be used for pain relief, and for severe pain, pethidine hydrochloride should be used ${ }^{[7]}$.

\subsection{Statistical methods}

The data was analyzed using Statistical Product and Service Solutions 20.0 software package. The measurement data of normal distribution was represented by $\mathrm{x} \pm \mathrm{s}$. The comparison between groups was performed by independent sample t-test, and the count data was assessed by 2 test. $P<$ 0.05 indicates significant difference.

\section{Results}

\subsection{Patient demographics and Pathologic findings}

The baseline demographics in the two groups were comparable, and there were no significant differences between the two groups $(P>0.05)$ (Table 1) .

Table 1 Patient demographics and Pathologic findings

\begin{tabular}{|c|c|c|c|c|c|}
\hline \multicolumn{2}{|c|}{ Characteristics } & $\begin{array}{l}\text { Experimental } \\
\text { Group }(\mathrm{n}=44)\end{array}$ & $\begin{array}{c}\text { Control } \\
\text { Group }(n=59)\end{array}$ & $t \& x^{2}$ & $P$ value \\
\hline \multicolumn{6}{|c|}{ Patient demographics } \\
\hline \multirow[t]{2}{*}{ Gender } & (Male/Female) & $24 / 20$ & $31 / 28$ & $x^{2}=0.041$ & $p=0.840$ \\
\hline & Age & $62.70 \pm 8.77$ & $61.98 \pm 8.36$ & $t=0.424$ & $p=0.672$ \\
\hline \multicolumn{2}{|c|}{ Body Mass Index } & $22.04 \pm 3.32$ & $22.46 \pm 3.05$ & $t=-0.682$ & $p=0.497$ \\
\hline \multicolumn{2}{|c|}{$\begin{array}{c}\text { Tumor location } \\
\text { (Up/Middle/Down) }\end{array}$} & $6 / 24 / 14$ & $11 / 27 / 21$ & $\chi^{2}=0.881$ & $p=0.644$ \\
\hline \multicolumn{2}{|c|}{ Smoking history(No/Yes) } & $20 / 24$ & $30 / 29$ & $x^{2}=0.293$ & $p=0.588$ \\
\hline \multicolumn{2}{|c|}{ Operation duration (min) } & $349.09 \pm 44.40$ & $348.81 \pm 44.53$ & $t=0.031$ & $p=0.975$ \\
\hline \multicolumn{2}{|c|}{ Retrieved Lymph nodes } & $13.11 \pm 2.33$ & $13.14 \pm 2.67$ & $t=0.044$ & $p=0.965$ \\
\hline \multicolumn{6}{|c|}{ Pathologic findings } \\
\hline \multicolumn{6}{|c|}{ Pathologic } \\
\hline \multicolumn{2}{|c|}{$\begin{array}{c}\text { types(adenocarcinoma/Squamo } \\
\text { us cell carcinomas) }\end{array}$} & $3 / 41$ & $5 / 54$ & $x^{2}=0.097$ & $p=0.756$ \\
\hline $\begin{array}{l}\text { Tumor } \\
\text { Node }\end{array}$ & $\mathrm{T} 1 / \mathrm{T} 2 / \mathrm{T} 3 / \mathrm{T} 4$ & $13 / 8 / 17 / 6$ & $13 / 12 / 26 / 8$ & $x^{2}=0.802$ & $p=0.849$ \\
\hline $\begin{array}{l}\text { Metasta } \\
\text { sis }\end{array}$ & $\mathrm{N} 0 / \mathrm{N} 1 / \mathrm{N} 2 / \mathrm{N} 3$ & $18 / 14 / 6 / 6$ & $31 / 15 / 7 / 6$ & $x^{2}=1.406$ & $p=0.704$ \\
\hline
\end{tabular}

2.2 Comparison of postoperative drainage effects between the two groups of patients with 


\section{esophageal cancer}

On the first day after operation, the difference of thoracic drainage volume of the two groups was not statistically significant $(P>0.05)$. Later on the 2 nd to 5 th day, the drainage volume in the central venous catheter group was significantly less than that of the conventional group $(P<0.05)$. Comparison of postoperative extubation time between the two groups showed no difference $(P>0.05)$. However, the postoperative hospital stay and drainage tube healing time between the two groups were significant different $(P<0.05)$. In regard to the postoperative complications, there are no different between the two groups $(P>0.05)$.

Table 2 Comparison of postoperative drainage effects between the two groups of patients with esophageal cancer

\begin{tabular}{|c|c|c|c|c|c|}
\hline \multicolumn{2}{|c|}{ Postoperative outcomes } & $\begin{array}{c}\text { Experimental } \\
\text { Group }(n=44)\end{array}$ & $\begin{array}{c}\text { Control } \\
\text { Group }(n=59)\end{array}$ & $\mathrm{t} \& \chi^{2}$ & $P$ value \\
\hline \multirow{5}{*}{$\begin{array}{c}\text { Drainage } \\
\text { volume(ml) }\end{array}$} & 1th & $547.72 \pm 64.66$ & $536.27 \pm 84.67$ & $t=0.749$ & 0.456 \\
\hline & 2th & $260.23 \pm 45.21$ & $342.54 \pm 51.91$ & $t=-8.404$ & 0.000 \\
\hline & 3th & $187.50 \pm 34.31$ & $308.47 \pm 38.45$ & $t=-16.527$ & 0.000 \\
\hline & 4th & $155.68 \pm 36.11$ & $255.93 \pm 44.61$ & $t=-12.214$ & 0.000 \\
\hline & 5th & $136.96 \pm 26.45$ & $158.84 \pm 28.39$ & $t=-46.927$ & 0.000 \\
\hline \multicolumn{2}{|c|}{ Extubation time(days) } & $7.61 \pm 0.92$ & $7.53 \pm 1.02$ & $t=0.452$ & 0.653 \\
\hline \multicolumn{2}{|c|}{$\begin{array}{l}\text { Postoperative hospital } \\
\text { stay(days) }\end{array}$} & $11.75 \pm 2.02$ & $13.03 \pm 1.29$ & $t=-3.926$ & 0.000 \\
\hline \multicolumn{2}{|c|}{$\begin{array}{l}\text { Drainage tube healing } \\
\text { time(days) }\end{array}$} & $5.64 \pm 1.12$ & $14.02 \pm 1.32$ & $t=-33.945$ & 0.000 \\
\hline \multicolumn{2}{|c|}{ Pleural effusion } & 2 & 11 & $x=3.354$ & 0.067 \\
\hline \multicolumn{2}{|c|}{$\begin{array}{c}\text { Reposition the tube after } \\
\text { extubation }\end{array}$} & 2 & 7 & $x=0.900$ & 0.343 \\
\hline \multicolumn{2}{|c|}{ Atelectasis } & 3 & 11 & $x=2.079$ & 0.149 \\
\hline \multicolumn{2}{|c|}{ Pneumothorax } & 1 & 5 & $x=0.817$ & 0.366 \\
\hline \multicolumn{2}{|c|}{$\begin{array}{c}\text { Subcutaneous } \\
\text { emphysema }\end{array}$} & 2 & 2 & $x=0.00$ & 1.000 \\
\hline \multicolumn{2}{|c|}{ Lung infection } & 2 & 7 & $x=0.900$ & 0.343 \\
\hline \multicolumn{2}{|c|}{ Anastomotic leakage } & 3 & 8 & $x=0.598$ & 0.439 \\
\hline
\end{tabular}

\subsection{Comparison of postoperative pain scores between the two groups of patients with esophageal cancer}

Next, the postoperative pain scores of the two groups were compared. As shown in Table 3, it turned out that the pain scores of the central venous catheter group were significantly lower than those of the conventional group $(P<0.05)$.

Table 3 Comparison of postoperative pain scores between two groups of esophageal cancer patients

\begin{tabular}{ccccc}
\hline $\begin{array}{c}\text { Postoperative } \\
\text { Pain score }\end{array}$ & Experimental & Control Group $(\mathrm{n}$ & $\mathrm{t}$ & \multirow{2}{*}{$P$ value } \\
\hline
\end{tabular}




\begin{tabular}{lllll}
\hline $12 \mathrm{~h}$ & $3.59 \pm 0.73$ & $5.14 \pm 0.79$ & $\mathrm{t}=-9.288$ & 0.000 \\
$24 \mathrm{~h}$ & $2.75 \pm 0.61$ & $3.51 \pm 0.60$ & $\mathrm{t}=-6.292$ & 0.000 \\
$48 \mathrm{~h}$ & $2.20 \pm 0.46$ & $3.22 \pm 0.56$ & $\mathrm{t}=-9.810$ & 0.000 \\
$72 \mathrm{~h}$ & $1.55 \pm 0.50$ & $2.59 \pm 0.62$ & $\mathrm{t}=-9.182$ & 0.000 \\
$96 \mathrm{~h}$ & $1.36 \pm 0.49$ & $2.46 \pm 0.50$ & $\mathrm{t}=-11.078$ & 0.000 \\
\hline
\end{tabular}

\section{Discussion}

Placing a closed thoracic drainage tube after esophageal cancer surgery can not only eliminate residual fluid and gas, promote lung lobe recruitment, and reduce the incidence of postoperative lung infections, it also serves as the "eye" of the surgeon to observe postoperative complications such as potential bleeding, chyle leakage, and anastomotic leakage ${ }^{[8]}$. However, the traditional concept believes that the thicker the diameter of the drainage tube, the better the drainage effect, so thicker closed chest drainage tube has been used till today. Initially, we studied the influence of the diameter of the drainage tube on the comfort of patients from the thoracoscopic lobectomy, and some scholars tried to use a urinary tube with satisfactory results ${ }^{[9,10]}$. Therefore, we use a thinner drainage tube to assist the chest drainage tube with a larger diameter after esophageal cancer surgery, resulting in significantly improved clinical effects ${ }^{[11]}$. Later, it was simplified by using a single negative pressure drainage ball for chest drainage, which greatly reduced postoperative chest pain ${ }^{[12]}$. There is a few literature ${ }^{[13]}$ reported that placement of no thoracic drainage tube but a mediastinal drainage after esophageal cancer surgery can greatly reduce the postoperative pain of the patient, but it is only suitable for resection of esophageal cancer through the mediastinal route, not for thoracoscopic esophageal cancer resection.

Central venous catheters have been widely used in the treatment of primary or secondary pleural effusion drainage and traumatic hemothorax ${ }^{[14]}$. At the meantime, the application has certain clinical value for the treatment of tuberculous pleurisy ${ }^{[15]}$. However, there are relatively few clinical studies on central venous catheters instead of traditional chest tubes currently. This may be due to concerns that the smaller diameter may affect postoperative observations such as postoperative bleeding, anastomotic leakage, chylothorax and other complications, and delays in treatment will endanger the lives of patients' safety. Retrospecting the esophageal surgery in our cancer medical center in the past ten years, the incidence of postoperative hemorrhage, anastomotic leakage ${ }^{[16,17]}$, and chylothorax ${ }^{[18]}$ are very low. In intrathoracic anastomosis of esophageal cancer surgery [19], if an anastomotic leakage occurs, it will cause chest and lung infections, leading extremely high fatality rate. Therefore, we always stick to the neck anastomosis in radical esophageal cancer surgery. Once an anastomotic leakage occurs, it will not be life-threatening due to chest infection, but only affects the healing of the neck incision and can be cured by irrigating the neck anastomosis.

In this study, the great advantage of central venous catheter thoracic drainage is less pleural irritation. The thoracic drainage volume of the two groups were recorded and analyzed on the 1st to 5th day after the operation. The data showed the drainage volume of the two groups on the first day were similar, which may be due to that the surgical wound exudation is the main factor of the chest drainage. On days 2 to 5 , surgical wound exudation slowly decreased or even stopped. At 
this time, the main factor that affects the thoracic drainage is the thicker chest tube diameter, which may strongly stimulate the pleura and intercostal nerves, and increase thoracic drainage. The central venous catheter had almost no stimulation to the pleura, so the drainage volume of the experimental group was significantly less than that of the control group, consequently causing less pain.

Furthermore, from the data the postoperative Numerical Rating Scale score, it can be clearly seen that the pain of the experimental group is significantly lower than that of the control group. The central venous catheter does not need a leading wire because of its small diameter, avoiding the pain of the drainage tube orifice caused by ligating the pre-wire. And it improves the quality of life of patients after surgery with less hospital stay. The central venous catheter in our study is connected to a disposable anti-reflux bag, so that there is no need to carry a bulky closed chest drainage bottle. As a result, it caused no pain or slight pain, which is conducive to coughing and sputum discharge, making it more convenient for patients to get out of bed and reducing the risk of postoperative thrombosis. The length of hospital stay in the experimental group was shorter than that in the control group, and the effect of rapid postoperative recovery was significant, which also conformed to the enhanced recovery after surgery concept of faster wound healing. It is also suggested in this study that the healing time of the experimental group was obviously much shorter than that of the control group. The thicker chest drainage tube was indwelled for a long time, resulting in redness and swelling of the tube orifice. After being pulled out, the wound healing time was longer and the scar was apparent. In addition, small diameter of the central venous catheter caused almost no pain after being pulled out, and the wound heals quickly.

Despite of the advantages above, the use of central venous catheters still has limitations: the fibrous cord formed by the postoperative drainage increased the risk of poor drainage or blockage in thin lumen. In this view, we can statistically analyze the approximate interval of thoracic drainage within $12 \mathrm{~h}, 24 \mathrm{~h}$, and $48 \mathrm{~h}$ after thoracoscopic esophageal cancer surgery, and use this as a reference. If the thoracic drainage volume is significantly less than the normal range, the risk of poor drainage or blockage should be highly alert of. Then bedside chest X-ray or bedside chest ultrasound and other analysis should be taken to search for the cause, which to be dealt with correctly and quickly. The soft material of the central venous catheter may at risk of being easy to fold after operation. The traditional chest tube is basically at right angles to the chest cavity. Because of its thick tube diameter, there is barely no postoperative folding. If we still follow the traditional way, the probability of postoperative folding will be significantly high. Therefore, the angle between the central venous catheter and the chest wall should be carefully adjusted to avoid folding.

\section{Conclusion}

In summary, the central venous catheter used for thoracic drainage is not only safe and reliable, but also greatly reduces the postoperative pain and discomfort of the patient, accelerating the postoperative recovery of the patient. Meanwhile, it conforms to the concept of enhanced recovery after surgery ${ }^{[20]}$ and is worthy of clinical application in the future.

\section{Declarations}

\section{Acknowledgements}


Not applicable.

\section{Disclosure of conflict of interest}

None.

\section{Authors' contributions}

Yang Zhao and Tao Li performed the surgery. Dong Song and Tao Wang assisted in the surgery. Zhi-Xia Bai performed statistical analysis and assisted with drafting of the manuscript. Yang Zhao wrote the manuscript. All authors read and approved the final manuscript.

\section{Funding}

This work was supported by the First-Class Discipline Construction Founded Project of NingXia Medical University and the School of Cincical Medicine [Grant No.NXYLXK2017A05].

\section{Availability of data and materials}

The datasets used and analyzed during the current study are available from the corresponding author on reasonable request.

\section{Ethics approval and consent to participate}

This study was approved by the Regional Ethics Committee of Ningxia Medical University General Hospital and all patients signed informed consents

\section{Consent for publication}

Not applicable.

\section{Competing interests}

The authors declare that they have no competing interests.

\section{References}

[1] Bollschweiler E, Plum P, Mönig SP, Hölscher AH. Current and future treatment options for esophageal cancer in the elderly. Expert Opin Pharmacother. 2017 Jul;18(10):1001-1010. doi: 10.1080/14656566.2017.1334764. Epub 2017 Jun 7. PMID: 28540761.

[2] Yibulayin W, Abulizi S, Lv H, Sun W. Minimally invasive oesophagectomy versus open esophagectomy for resectable esophageal cancer: a meta-analysis. World J Surg Oncol. 2016 Dec 8;14(1):304. doi: 10.1186/s12957-016-1062-7. PMID: 27927246; PMCID: PMC5143462.

[3] Berlth F, Plum PS, Chon SH, Gutschow CA, Bollschweiler E, Hölscher AH. Total minimally invasive esophagectomy for esophageal adenocarcinoma reduces postoperative pain and pneumonia compared to hybrid esophagectomy. Surg Endosc. 2018 Dec;32(12):4957-4965. doi: 10.1007/s00464-018-6257-2. Epub 2018 Jun 21. PMID: 29931451.

[4] Jínek T, Adamcik L, Duda M, Skrovina M. Hybrid thoracoscopic esophagectomy for cancer retrospective analysis and comparison with transhiatal resection. Rozhl Chir. 2018 Spring;97(7):320-327. English. PMID: 30442013.

[5] Rubinkiewicz M, Witowski J, Su M, Major P, Pędziwiatr M. Enhanced recovery after surgery (ERAS) programs for esophagectomy. J Thorac Dis. 2019 Apr;11(Suppl 5):S685-S691. doi: 10.21037/jtd.2018.11.56. PMID: 31080645; PMCID: PMC6503272.

[6] Ashok A, Niyogi D, Ranganathan P, Tandon S, Bhaskar M, Karimundackal G, Jiwnani S, Shetmahajan M, Pramesh CS. The enhanced recovery after surgery (ERAS) protocol to promote recovery following esophageal cancer resection. Surg Today. 2020 Apr;50(4):323-334. doi: 10.1007/s00595-020-01956-1. Epub 2020 Feb 11. Erratum in: Surg Today. 2020 Mar 25;: PMID: 32048046; PMCID: PMC7098920.

[7] Jin SJ, Park JY, Kim DH, Yoon SH, Kim E, Hwang JH, Song C, Kim YK. Comparison of 
postoperative pain between laparoscopic and robot-assisted partial nephrectomies for renal tumors: A propensity score matching analysis. Medicine (Baltimore). 2017 Jul;96(29):e7581. doi: 10.1097/MD.0000000000007581. PMID: 28723795; PMCID: PMC5521935.

[8] Wan J, Che Y, Kang N, Zhang R. Surgical Method, Postoperative Complications, and Gastrointestinal Motility of Thoraco-Laparoscopy 3-Field Esophagectomy in Treatment of Esophageal Cancer. Med Sci Monit. 2016 Jun 16;22:2056-65. doi: 10.12659/msm.895882. PMID: 27310399; PMCID: PMC4913812.

[9] Lai Y, Wang X, Zhou H, Kunzhou PL, Che G. Is it safe and practical to use a Foley catheter as a chest tube for lung cancer patients after lobectomy? A prospective cohort study with 441 cases. Int J Surg. 2018 Aug;56:215-220. doi: 10.1016/j.jjsu.2018.06.028. Epub 2018 Jun 21. PMID: 29936194.

[10] Yang M, Fan J, Zhou H, Du H, Qiu F, Lin L, Liu L, Li W, Che G. [What are the Advantages? A Prospective Analysis of 16 versus 28 French Chest Tube Sizes in Video-assisted Thoracoscopic Surgery Lobectomy of Lung Cancer]. Zhongguo Fei Ai Za Zhi. 2015 Aug;18(8):512-7. Chinese. doi: 10.3779/j.issn.1009-3419.2015.08.08. PMID: 26302349; PMCID: PMC6000231.

[11] Nakanishi R, Fujino Y, Kato M, Miura T, Yasuda M, Oda R, Yokota K, Okuda K, Haneda H. Early chest tube removal after thoracoscopic lobectomy with the aid of an additional thin tube: a prospective multi-institutional study. Gen Thorac Cardiovasc Surg. 2018 Dec;66(12):723-730. doi: 10.1007/s11748-018-0993-z. Epub 2018 Aug 21. PMID: 30132222.

[12] Cui Z, Zhang Y, Xu C, Ding C, Chen J, Li C, Zhao J. Comparison of the results of two chest tube managements during an enhanced recovery program after video-assisted thoracoscopic lobectomy: A randomized trial. Thorac Cancer. 2019 Oct;10(10):1993-1999. doi: 10.1111/1759-7714.13183. Epub 2019 Sep 2. PMID: 31475791; PMCID: PMC6775025.

[13] Cai L, Li Y, Wang WB, Guo M, Lian X, Xiao SA, Xu GH, Yang XW, Sun L, Zhang HW. Is closed thoracic drainage tube necessary for minimally invasive thoracoscopic-esophagectomy? $\mathrm{J}$ Thorac Dis. 2018 Mar;10(3):1548-1553. doi: 10.21037/jtd.2018.02.59. PMID: 29707305; PMCID: PMC5906305.

[14] Yi JH, Liu HB, Zhang M, Wu JS, Yang JX, Chen JM, Xu SX, Wang JA. Management of traumatic hemothorax by closed thoracic drainage using a central venous catheter. J Zhejiang Univ Sci B. 2012 Jan;13(1):43-8. doi: 10.1631/jzus.B1100161. PMID: 22205619; PMCID: PMC3251751.

[15] Song L, Zhang Y, Jia Q. Central Venous Catheter Based Closed Thoracic Drainage in the Treatment of Tuberculous Pleuritis. Pak J Med Sci. 2019 Jul-Aug;35(4):1024-1029. doi: 10.12669/pjms.35.4.63. PMID: 31372136; PMCID: PMC6659076.

[16] Shuto K, Kono T, Akutsu Y, Uesato M, Mori M, Matsuo K, Kosugi C, Hirano A, Tanaka K, Okazumi S, Koda K, Matsubara H. Naso-esophageal extraluminal drainage for postoperative anastomotic leak after thoracic esophagectomy for patients with esophageal cancer. Dis Esophagus. 2017 Feb 1;30(3):1-9. doi: 10.1111/dote.12492. PMID: 27862613.

[17] Guo W, Zhu L, Wu Y, Yang S, Du H, Zhou X, Che J, Hang J, Li H. Endoscope-assisted mediastinal drainage therapy for anastomosis leakage after esophagectomy: a retrospective cohort study. Ann Transl Med. 2019 Dec;7(23):747. doi: 10.21037/atm.2019.11.103. PMID: 32042763; PMCID: PMC6989978.

[18] Lambertz R, Chang DH, Hickethier T, Bagheri M, Leers JM, Bruns CJ, Schröder W. Ultrasound-guided lymphangiography and interventional embolization of chylous leaks following 
esophagectomy. Innov Surg Sci. 2019 Mar 9;4(3):85-90. doi: 10.1515/iss-2018-0037. PMID: 31709299; PMCID: PMC6817730.

[19] van Rossum PSN, Haverkamp L, Carvello M, Ruurda JP, van Hillegersberg R. Management and outcome of cervical versus intrathoracic manifestation of cervical anastomotic leakage after transthoracic esophagectomy for cancer. Dis Esophagus. 2017 Jan 1;30(1):1-8. doi: 10.1111/dote.12472. PMID: 26919029.

[20] Li Y. [Strategy and prospective of enhanced recovery after surgery for esophageal cancer]. Zhonghua Wei Chang Wai Ke Za Zhi. 2016 Sep 25;19(9):965-970. Chinese. PMID: 27680060. 


\section{Figures}

\section{Figure 1}

The single lumen central venous catheter $(5 \mathrm{~F})$ with the diameter of $1.6 \mathrm{~mm}$; $\mathrm{B}$, The silicone tube $(16 \mathrm{~F})$ with the diameter of $5.2 \mathrm{~mm} ; \mathrm{C}, \mathrm{D}$, The single central venous catheter and the silicone tube drainage were used respectively after esophageal cancer surgery.

\section{Supplementary Files}

This is a list of supplementary files associated with this preprint. Click to download.

- CAREchecklist.pdf 